\title{
Strongly localized plasmon oscillations in a cluster of two metallic nanospheres and their influence on spontaneous emission of an atom
}

\author{
V.V. Klimov and D.V. Guzatov \\ P.N. Lebedev Physical Institute, Russian Academy of Sciences, \\ 53 Leninsky Prospekt, 119991 Moscow, Russia
}

August 16, 2018

\begin{abstract}
The plasmon oscillations in a cluster of two metallic nanospheres are studied theoretically. Particular attention is paid to the case of nearly touching spheres. Simple analytical expressions have been found for the spectra of plasmon oscillations of different symmetry in this case. A new type of the plasmon oscillations, which are strongly localized between the spheres, and which totally disappear at separation of the spheres, has been discovered. The found plasmon oscillations have a dramatic effect on optical properties of an atom localized between the spheres.
\end{abstract}

Much attention has been paid, of late, to the experimental and theoretical study of optical properties of the metallic nanoparticles. This interest is mainly due to a considerable enhancement of local fields near the nanoparticles. An especially high increase occurs in the case of plasmon polariton resonances ("plasmons") [1, 2] or phonon polariton resonances ("phonons") 3]. On the basis of this effect one considers quite a number of possible applications. One of the most developed is the use of large local fields for enhancement of the Raman scattering cross-section [4. Recent experiments have shown that such an increase may achieve 10-14 orders of magnitude, which may help to resolve separate molecules [5]- 7 . The local enhancement of the fields can also be used to increase the fluorescence intensity and to determine the structure of a single DNA strand without using the fluorescent labels [8, 9]. By using the nanoparticles of complex configuration one can provide enhancement of both the absorption and the emission of light by natural and artificial fluorophores [10. Of particular interest and promise are the studies of optical properties of the clusters of two and more metallic nanoparticles, because by changing the cluster's geometry one can effectively control the spectra of the plasmon oscillations. This effect makes it possible to produce, for example, new types of biosensors [11-[13. A whole series of experimental [14-[17] and theoretical [18]- 27] studies have been devoted to the two-particle clusters. 
Among the theoretical works one should note the paper [18, where the method for defining the plasmon oscillations of a two-sphere cluster on the basis of a plasmon hybridization of separate spheres was proposed.

Most interesting is the case of nearly touching particles where the enhancement of local fields is particularly high. This is the most complicated case as well. In 14-17 this interesting case was not actually considered, and the paper 19] being especially devoted to that problem, turned out to be misleading. Basing on the numerical consideration of one of the particular cases, the conclusion was made in [20] about failure of the Mie theory for the closely set nanospheres.

In the present work we will investigate the most interesting and the most complicated case of the plasmon (phonon) oscillations in the cluster of two almost touching metallic nanospheres. We shall derive analytical expressions for the plasmon spectra in this region. It will be shown that in this system there exists a new type of plasmon oscillations (plasmonic molecule), which is strongly localized between the spheres, and which cannot be found within the framework of the hybridization method [18. We shall also show that new modes can be excited only by a localized light source (an excited atom, molecule, or a quantum dot) that should lead to a dramatic changing of optical properties of an atom. A geometry of the problem is illustrated in Fig.1. In the case of $\varepsilon_{1}<0$, $\varepsilon_{2}=1$ the geometry corresponds to two metallic nanoparticles, and in the case of $\varepsilon_{1}=1, \varepsilon_{2}<0$ to two spherical cavities (bubbles) in a metal. Hereinafter we assume that the dimensions of the studied particles are sufficiently small in order one could use the longwavelength perturbation theory 28 but at the same time, they are essentially large for one could neglect the non-local effects (space dispersion of permittivity) [29]. For simplicity here we will consider only the case of equal nanospheres.

To determine the spectrum of the plasmon oscillations in the first order of longwavelength perturbation theory [28] one must solve the equations:

$$
\operatorname{div} \mathbf{D}=0, \quad \operatorname{rot} \mathbf{E}=0
$$

with usual boundary conditions of continuity of the tangential component of $\mathbf{E}$ and the normal component of $\mathbf{D}$. To find further small corrections to spectrum of plasmon oscillations one must use procedure elaborated in 28. By means of a conventional substitution, $\mathbf{E}=-\nabla \Phi$, the system of equations (11) is reduced to the Laplace equation for the potential $\Phi$, which can be conveniently solved in a bispherical system of coordinates. The bispherical coordinates $(-\infty<\eta<\infty$, $0<\xi \leq \pi, 0 \leq \varphi<2 \pi)$ are connected with the Cartesian coordinates by the relations 30

$$
\begin{aligned}
& x=a \frac{\sin \xi \cos \varphi}{\cosh \eta-\cos \xi} \\
& y=a \frac{\sin \xi \sin \varphi}{\cosh \eta-\cos \xi} \\
& z=a \frac{\sinh \eta}{\cosh \eta-\cos \xi}
\end{aligned}
$$


The surface $\eta=-\eta_{0}<0$ is the sphere of the radius $R_{0}=a / \sinh \eta_{0}$. The other sphere can be set in the similar way by the equality $\eta=\eta_{0}>0$. The dimensional constant $a$ is the half distance between the poles of a bispherical system of coordinates, and is determined by the next expression: $a=\sqrt{R_{12}^{2} / 4-R_{0}^{2}}$, where $R_{12}$ is the distance between the centers of the first and second spheres.

The potential in space between the spheres $\left(-\eta_{0}<\eta<\eta_{0}\right)$ can be written in the form

$$
\begin{aligned}
\Phi & =\frac{1}{a} \sqrt{\cosh \eta-\cos \xi} \sum_{n=0}^{\infty} \sum_{m=0}^{n} P_{n}^{m}(\cos \xi) \\
& \left\{\left(\alpha_{m n} \cos (m \varphi)+\beta_{m n} \sin (m \varphi)\right) \cosh \left(\left(n+\frac{1}{2}\right) \eta\right)\right. \\
& \left.+\left(\gamma_{m n} \cos (m \varphi)+\delta_{m n} \sin (m \varphi)\right) \sinh \left(\left(n+\frac{1}{2}\right) \eta\right)\right\}
\end{aligned}
$$

where $\alpha_{m n}, \beta_{m n}, \gamma_{m n}, \delta_{m n}$ are the coefficients to be determined.

For the potential inside the nanospheres we have, respectively, $\left(\eta<-\eta_{0}<0\right)$

$$
\begin{aligned}
\Phi^{(1)}= & \frac{1}{a} \sqrt{\cosh \eta-\cos \xi} \sum_{n=0}^{\infty} \sum_{m=0}^{n} e^{\left(n+\frac{1}{2}\right) \eta} P_{n}^{m}(\cos \xi) \\
& \left\{a_{m n}^{(1)} \cos (m \varphi)+b_{m n}^{(1)} \sin (m \varphi)\right\}
\end{aligned}
$$

and $\left(\eta>\eta_{0}>0\right)$

$$
\begin{aligned}
\Phi^{(2)}= & \frac{1}{a} \sqrt{\cosh \eta-\cos \xi} \sum_{n=0}^{\infty} \sum_{m=0}^{n} e^{-\left(n+\frac{1}{2}\right) \eta} P_{n}^{m}(\cos \xi) \\
& \left\{a_{m n}^{(2)} \cos (m \varphi)+b_{m n}^{(2)} \sin (m \varphi)\right\}
\end{aligned}
$$

in (4) and (5) $a_{m n}^{(1)}, b_{m n}^{(1)}$ and $a_{m n}^{(2)}, b_{m n}^{(2)}$ are the coefficients to be determined.

Expressions for the potentials in the form of (3)-(5) have the most general form, and make it possible to determine all the modes irrespective of their symmetry.

By using usual boundary conditions at the surface of each of the nanospheres

$$
\begin{aligned}
\Phi\left(\eta=-\eta_{0}\right) & =\Phi^{(1)}\left(\eta=-\eta_{0}\right), \\
\varepsilon_{2} \frac{\partial}{\partial \eta} \Phi\left(\eta=-\eta_{0}\right) & =\varepsilon_{1} \frac{\partial}{\partial \eta} \Phi^{(1)}\left(\eta=-\eta_{0}\right)
\end{aligned}
$$

and 


$$
\begin{aligned}
\Phi\left(\eta=\eta_{0}\right) & =\Phi^{(1)}\left(\eta=\eta_{0}\right), \\
\varepsilon_{2} \frac{\partial}{\partial \eta} \Phi\left(\eta=\eta_{0}\right) & =\varepsilon_{1} \frac{\partial}{\partial \eta} \Phi^{(2)}\left(\eta=\eta_{0}\right)
\end{aligned}
$$

and the recurrent relations for the Legendre functions one can derive an infinite system of equations for all the coefficients: $\alpha_{m n}, \gamma_{m n}, \beta_{m n}, \delta_{m n}, a_{m n}$, and $b_{m n}$. In the most interesting case of the two identical nanospheres these equations can be written down in the following form

$$
\begin{aligned}
& \tau\left\{(n-m) \tilde{\alpha}_{m, n-1}+\left(\sinh \eta_{0}-(2 n+1) \cosh \eta_{0}\right) \tilde{\alpha}_{m n}\right. \\
& \left.+(n+m+1) \tilde{\alpha}_{m, n+1}\right\} \\
= & -(n-m) \tanh \left(\left(n-\frac{1}{2}\right) \eta_{0}\right) \tilde{\alpha}_{m, n-1} \\
& +\cosh \eta_{0}\left(\tanh \eta_{0}+(2 n+1) \tanh \left(\left(n+\frac{1}{2}\right) \eta_{0}\right)\right) \tilde{\alpha}_{m n} \\
& -(n+m+1) \tanh \left(\left(n+\frac{3}{2}\right) \eta_{0}\right) \tilde{\alpha}_{m, n+1}
\end{aligned}
$$

and

$$
\begin{aligned}
& \tau\left\{(n-m) \tilde{\gamma}_{m, n-1}+\left(\sinh \eta_{0}-(2 n+1) \cosh \eta_{0}\right) \tilde{\gamma}_{m n}\right. \\
& \left.+(n+m+1) \tilde{\gamma}_{m, n+1}\right\} \\
= & -(n-m) \operatorname{coth}\left(\left(n-\frac{1}{2}\right) \eta_{0}\right) \tilde{\gamma}_{m, n-1} \\
& +\cosh \eta_{0}\left(\tanh \eta_{0}+(2 n+1) \operatorname{coth}\left(\left(n+\frac{1}{2}\right) \eta_{0}\right)\right) \tilde{\gamma}_{m n} \\
& -(n+m+1) \operatorname{coth}\left(\left(n+\frac{3}{2}\right) \eta_{0}\right) \tilde{\gamma}_{m, n+1}
\end{aligned}
$$

where $\tau=\varepsilon_{1} / \varepsilon_{2}$ and $\tilde{\alpha}_{m n}=\alpha_{m n} \cosh \left(\left(n+\frac{1}{2}\right) \eta_{0}\right), \tilde{\gamma}_{m n}=\gamma_{m n} \sinh \left(\left(n+\frac{1}{2}\right) \eta_{0}\right)$. Note that the recurrent equations for the second series of the coefficients $\beta_{m n}$ and $\delta_{m n}$ have the form (8) and (9) (the substitutions $\alpha_{m n} \rightarrow \beta_{m n}$ and $\gamma_{m n} \rightarrow$ $\delta_{m n}$ are to be made).

The coefficients $a_{m n}^{(1)}$ and $a_{m n}^{(2)}$ for the fields inside the nanospheres can be found from the relations

$$
\begin{aligned}
& a_{m n}^{(1)} e^{-\left(n+\frac{1}{2}\right) \eta_{0}}=\tilde{\alpha}_{m n}-\tilde{\gamma}_{m n}, \\
& a_{m n}^{(2)} e^{-\left(n+\frac{1}{2}\right) \eta_{0}}=\tilde{\alpha}_{m n}+\tilde{\gamma}_{m n}
\end{aligned}
$$


The equations for the coefficients $b_{m n}^{(1)}$ and $b_{m n}^{(2)}$ can be derived from (10) by means of the following substitutions $a_{m n}^{(1)} \rightarrow b_{m n}^{(1)}, a_{m n}^{(2)} \rightarrow b_{m n}^{(2)}$, and $\alpha_{m n} \rightarrow \beta_{m n}$, $\gamma_{m n} \rightarrow \delta_{m n}$.

The infinite systems of homogeneous equations (8) and (9) will have nonzero solution only for special (resonant) values of $\tau=\varepsilon_{1} / \varepsilon_{2}$. To find these resonant values one should solve the generalized eigenvalue problem. Note that the coefficients $\alpha_{m n}$ and $\gamma_{m n}$ (which correspond to solution of different parity in $\eta$ ) enter into (8) and (9) independently.

Figure 2 illustrates the dependence of the resonant permittivity on distance between spheres for $m=1$. For any other $m$ we have similar spectra. One can see in Fig.2 that there are three types of the modes: $L-, M$ - and $T$-types. As seen in Fig.2, if the distance between the nanospheres increases the resonant permittivity for the $T$ - and $L$-modes takes the known [23, 24] values $\tau=-\frac{n+1}{n}$, which correspond to the plasmon oscillations of different electric multipolarity excited in a single sphere: $n=1$ corresponds to the dipole oscillations, $n=2$ corresponds to the quadrupole oscillations and so on. Such modes have been well studied and are described by hybridization of modes of the separate spheres [18. As for the $M$-modes, it seems that their appearance had not been observed earlier. These modes have a strongly localized character, because they can exist in the clusters with $\frac{R_{12}}{2 R_{0}} \leq 1.2$ only. Such modes can be considered as bound states of plasmons of the different spheres (plasmonic molecule). In order to excite such modes one needs a localized source of electromagnetic field. In the papers 24, 27 studying the surface modes of a cluster one did not observe the appearance of such modes because they considered an excitation of a cluster by a plane electromagnetic wave which can not excite new $M$-modes.

From the above Figure 2 it is seen that the behavior of the resonant permittivity in the region of the nearly touching spheres becomes very complicated. To describe that complex behavior we consider (8) at the limit of $\eta_{0} \rightarrow 0\left(\frac{R_{12}}{2 R_{0}} \rightarrow 1\right)$. In this case we have found an asymptotic expression for the resonance parameter $\tau$ (relative permittivity).

For the $M$-modes we have

$$
\tau_{m, M}=\frac{\varepsilon_{1}}{\varepsilon_{2}}=-\left(M+\delta_{m}\right) \operatorname{arccosh} \frac{R_{12}}{2 R_{0}}+\ldots
$$

where $m=0,1,2, \ldots ; M=1,2,3, \ldots ; \delta_{0}=1 / 2$ and $\delta_{1} \approx 0.914$.

For the $L$-modes one can obtain the following analytical solution

$$
\tau_{m, L}=\frac{\varepsilon_{1}}{\varepsilon_{2}}=-\left(m+L-\frac{1}{2}\right)^{-1} / \operatorname{arccosh} \frac{R_{12}}{2 R_{0}}+\ldots
$$

where $m=0,1,2, \ldots ; L=1,2,3, \ldots$ Of course expressions (11) and (12) are in agrement with Fig. 2 in the region $\frac{R_{12}}{2 R_{0}} \rightarrow 1$.

From these expressions it follows that the resonant relative permittivity of the cluster $\tau$ tends to zero ( $M$-modes) or to the infinity ( $L$-modes) if the nanospheres are drawing together. Tending of the relative permittivity to zero corresponds to two physically different cases, namely, two metallic nanospheres 
$\left(\varepsilon_{1}=0, \varepsilon_{2}=1\right)$ or two cavities in a metal $\left(\varepsilon_{1}=1, \varepsilon_{2} \rightarrow-\infty\right)$. The analogous situation occurs when the relative permittivity tends to the infinity.

The eigenvectors of the $L$ - and $M$-modes correspond to the resonant permittivity (12) and (11) at the limit of the closely set spheres can be calculated analytically as well,

$$
\left.\tilde{\alpha}_{0, M}=\frac{1}{M}\left(\begin{array}{c}
-1 \\
\vdots \\
-1
\end{array}\right\} M\right), \quad \tilde{\gamma}_{m, L}=\left(\begin{array}{c}
0 \\
\vdots \\
0 \\
0 \\
0
\end{array}\right) L-1
$$

Note that the analogous expression for $\tilde{\delta}_{m, L}$ coincides with expression (13) for $\tilde{\gamma}_{m, L}$, and $\tilde{\beta}_{0, M}=0$. One can substitute these vectors into (3)-(5) to find the expressions for the potential eigenfunctions.

For the eigenfunctions of the $M$-mode $(m=0)$ in space between the spheres we obtain

$$
\begin{aligned}
\left.\Phi_{0, M}\right|_{\eta_{0} \rightarrow 0} & \approx-\frac{1}{M a} \sqrt{\cosh \eta-\cos \xi} \sum_{n=0}^{M-1} \cosh \left(\left(n+\frac{1}{2}\right) \eta\right) P_{n}(\cos \xi) \\
& +\frac{1}{a} \sqrt{\cosh \eta-\cos \xi} \cosh \left(\left(M+\frac{1}{2}\right) \eta\right) P_{M}(\cos \xi)
\end{aligned}
$$

Inside the first sphere we have, correspondingly,

$$
\begin{aligned}
\left.\Phi_{0, M}^{(1)}\right|_{\eta_{0} \rightarrow 0} & \approx-\frac{1}{M a} \sqrt{\cosh \eta-\cos \xi} \\
& \sum_{n=0}^{M-1}\left(1+\left(n+\frac{1}{2}\right) \eta_{0}\right) e^{\left(n+\frac{1}{2}\right) \eta} P_{n}(\cos \xi) \\
& +\frac{1}{a} \sqrt{\cosh \eta-\cos \xi}\left(1+\left(M+\frac{1}{2}\right) \eta_{0}\right) e^{\left(M+\frac{1}{2}\right) \eta} P_{M}(\cos \xi)
\end{aligned}
$$

Inside the second sphere we obtain the analogous expression.

For the eigenfunctions of the $L$-mode in space between the spheres and inside the first sphere we have, correspondingly,

$$
\begin{aligned}
\left.\Phi_{m, L}\right|_{\eta_{0} \rightarrow 0} \approx & \frac{1}{a} \sqrt{2(\cosh \eta-\cos \xi)} \sinh \left(\left(L-\frac{1}{2}\right) \eta\right) \\
& P_{L-1}^{m}(\cos \xi) \sin \left(m \varphi+\frac{\pi}{4}\right)
\end{aligned}
$$


and

$$
\begin{aligned}
\left.\Phi_{m, L}^{(1)}\right|_{\eta_{0} \rightarrow 0} \approx & -\frac{\eta_{0}}{a}\left(L-\frac{1}{2}\right) \sqrt{2(\cosh \eta-\cos \xi)} e^{\left(L-\frac{1}{2}\right) \eta} \\
& P_{L-1}^{m}(\cos \xi) \sin \left(m \varphi+\frac{\pi}{4}\right)
\end{aligned}
$$

Inside the second sphere we get the analogous expression.

Knowing the eigenfunctions one can calculate a surface charge $\sigma^{(i)}$ that is stored on each of the nanospheres $(i=1,2)$

$$
\sigma^{(i)}=\left.\frac{\tau-1}{4 \pi a}\left(\cosh \eta_{0}-\cos \xi\right)(-1)^{i} \frac{\partial \Phi^{(i)}}{\partial \eta}\right|_{\eta=\eta_{i}}
$$

Using the asymptotic expressions for the potentials (14)-(17) one can write down the expression for the charge for the $M$-mode $(m=0)$ in the form

$$
\begin{aligned}
\sigma_{0, M}^{(1)}= & \sigma_{0, M}^{(2)} \approx \frac{(1-\cos \xi)^{3 / 2}}{8 \pi a^{2}} \\
& \left(-\frac{1}{M} \sum_{n=0}^{M-1}(2 n+1) P_{n}(\cos \xi)+(2 M+1) P_{M}(\cos \xi)\right)
\end{aligned}
$$

For the $L$-mode we get, respectively,

$$
\sigma_{m, L}^{(1)}=-\sigma_{m, L}^{(2)} \approx \frac{(1-\cos \xi)^{3 / 2}}{4 \sqrt{2} \pi a^{2}} \frac{(2 L-1)^{2}}{2(m+L)-1} P_{L-1}^{m}(\cos \xi) \sin \left(m \varphi+\frac{\pi}{4}\right)
$$

We did not succeed in finding the analytical solution for the $T$-mode. Moreover, it is difficult to find limit at $\frac{R_{12}}{2 R_{0}} \rightarrow 1$ of these curves. To investigate this limit let us consider the limiting case of the touching spheres $\left(\frac{R_{12}}{2 R_{0}}=1\right)$ within the coordinate system of the touching spheres [31. In this case, the spectrum of the plasmon oscillations that are symmetrical to the $z=0$ plane is determined by the integral equation

$$
\begin{aligned}
& K_{m}\left(\lambda^{\prime}\right) \int_{0}^{\lambda^{\prime}} d \lambda A_{m \lambda} I_{m}(\lambda)+I_{m}\left(\lambda^{\prime}\right) \int_{\lambda^{\prime}}^{\infty} d \lambda A_{m \lambda} K_{m}(\lambda) \\
= & \frac{1}{\tau-1}\left(\tau+\tanh \left(\lambda^{\prime}\right)\right) A_{m \lambda^{\prime}}
\end{aligned}
$$

where $I_{m}$ and $K_{m}$ are the modified Bessel function and the Macdonald function, and $A_{m \lambda}$ is the Hankel transform of the potential eigenfunction.

The integral equation (21) for the eigenvalues $\tau_{m, T}$ can be solved numerically 


$$
\begin{aligned}
& \tau_{0,1} \approx-1.696, \quad \tau_{0,2} \approx-1.355, \quad \tau_{0,3} \approx-1.237, \\
& \tau_{1,1} \approx-1.799, \quad \tau_{1,2} \approx-1.386, \quad \tau_{1,3} \approx-1.182
\end{aligned}
$$

Thus, in the case of the touching nanospheres the intrinsic resonant values of $T$-modes lie within the interval $[-2,-1]$. The numerical solution of (22) agrees well with the functions represented in Fig. 2 in the region of $\frac{R_{12}}{2 R_{0}} \rightarrow 1$ ( $T$-modes).

In the case of antisymmetrical (longitudinal) $L$-modes the integral equation has the form:

$$
\begin{aligned}
& K_{m}\left(\lambda^{\prime}\right) \int_{0}^{\lambda^{\prime}} d \lambda A_{m \lambda} I_{m}(\lambda)+I_{m}\left(\lambda^{\prime}\right) \int_{\lambda^{\prime}}^{\infty} d \lambda A_{m \lambda} K_{m}(\lambda) \\
= & \frac{1}{\tau-1}\left(\tau+\operatorname{coth}\left(\lambda^{\prime}\right)\right) A_{m \lambda^{\prime}}
\end{aligned}
$$

which seems to have only trivial solutions at finite $\varepsilon_{1} / \varepsilon_{2}$. This result agrees with the asymptotic of (12) and disagrees with a numerical solution found in [20. Perhaps, its difference from [20] is due to insufficient accuracy of the calculations in 20].

Figure 3 illustrates surface charge distributions derived from an accurate numerical solution of the recurrent equations (8) and (9) for $m=1$. For any other $m$ we have the similar distributions. To plot these distributions we have used a polar system of coordinates $(\rho, \theta)$ for each sphere in plane $y=0$. The surface charge distribution was depicted with $\rho=R_{0}(1+\tilde{\sigma}(\theta))$ polar curve where $\tilde{\sigma}(\theta)$ is the dimensionless surface charge density as a function of polar angle of corresponding sphere. The $M$-mode and its vividly local character are of most interest. This mode is actually localized in the region with characteristic size of the order of size of the gap between the nanospheres. The $T$ - and $L$ modes are due to the charge oscillations over the whole surface of spheres and are analogous to the charge distribution on separate spheres. Note that the $L$-modes have also appreciable localization between the spheres.

It is very important that new $M$ - modes can not be excited by uniform electric field. To excite such modes one must use nonuniform field such as dipole radiation of an atom or molecule placed near the cluster. On the other hand, the excited plasmon modes will influence on decay rate and fluorescence of an atom or molecule. The modification of decay rate by new modes can be used to detect them.

The radiative decay rate $\gamma^{\text {radiative }}$ of an atom placed near any nanobody can be described by 32, 33.

$$
\frac{\gamma^{\text {radiative }}}{\gamma_{0}}=\frac{\left|\mathbf{d}_{\text {total }}\right|^{2}}{d_{0}^{2}}
$$

where $\gamma_{0}$ is the atom spontaneous decay rate in free space; $\mathbf{d}_{0}, \mathbf{d}_{\text {total }}=\mathbf{d}_{0}+\delta \mathbf{d}$, the dipole transition moment and the total dipole moment of an atom + cluster, 
respectively. In our case the expression for the dipole moment induced in the cluster takes the form 34

$$
\begin{aligned}
& \delta d_{x}=-\sqrt{2} a \sum_{n=1}^{\infty} n(n+1)\left(\mathbf{d}_{0} \nabla^{\prime}\right) \alpha_{1 n}, \\
& \delta d_{y}=-\sqrt{2} a \sum_{n=1}^{\infty} n(n+1)\left(\mathbf{d}_{0} \nabla^{\prime}\right) \beta_{1 n}, \\
& \delta d_{z}=\sqrt{2} a \sum_{n=0}^{\infty}(2 n+1)\left(\mathbf{d}_{0} \nabla^{\prime}\right) \gamma_{0 n}
\end{aligned}
$$

where $\nabla^{\prime}$ means gradient over the atom's coordinates; $\alpha_{1 n}, \beta_{1 n}, \gamma_{0 n}$ are the coefficients in (3) found with taking into account the atomic dipole source.

Figure 4 illustrates the dependence of the spontaneous decay rate of an atom near two nanospheres (24), (25) on a transition wavelength. The spheres are placed in vacuum $\left(\varepsilon_{2}=1\right)$ and made of $\mathrm{SiC}$, where phonon resonances can be excited in the infrared [35]. Strictly speaking $\mathrm{SiC}$ is uniaxial hexagonal crystal with anisotropic tensor of dielectric permittivity. However, strong TO $\perp(797$ $\left.\mathrm{cm}^{-1}\right)$ and $\mathrm{TO} \|\left(788 \mathrm{~cm}^{-1}\right)$ modes have very similar dielectric properties. It allows us to consider $\mathrm{SiC}$ crystal in the first approximation as isotropic one in the region of 788-797 $\mathrm{cm}^{-1}$. In Fig.4 the influence of these modes on spontaneous emission of an atom is shown. As seen in Fig.4(a), the $T$ - and $M$-modes can be excited by the atom with the transversal dipole (the atom lies precisely between the spheres). The excitation of only these modes can be explained by the symmetry of an induced charge at the surface of the spheres, in respect to the plane with $z=0$. As the real part of the permittivity increases (decrease in the transition wavelength) then $T$-modes are replaced by $M$-modes, in accordance with the functions of Fig.2, at transition over the $\varepsilon_{1}=-1$ point. It is also important to mention that the spontaneous decay rate for the $M$-modes is substantially higher than that for the $T$-modes. In Fig.4(b) one can observe only the $L$-modes in the spontaneous decay rate spectrum, provided that the dipole moment of an atom is oriented along $z$-axis (the atom lies precisely between the spheres). This fact is due to the symmetry of the excitation source also, i.e. the charge distribution on spheres is to be antisymmetrical to the plane with $z=0$. In the same Figure one can clearly see an increase in the number of the resonances as real part of the permittivity approaches $\varepsilon_{1}=-1$, which agrees with the functions represented in Fig.2. As the transition wavelength decreases (going over $\varepsilon_{1}=-1$ ) no resonant phenomena are observed for such an orientation of the dipole, except for the dip at $\varepsilon_{1}=0$.

Thus, in this paper the spectrum of plasmon oscillations occurring in a cluster of two closely set nanospheres, or spherical bubbles, has been considered in detail, and the respective analytical expressions have been found. A new type of strongly localized plasmons oscillations has been discovered. Such modes can be considered as bound states of plasmons of the different spheres (plasmonic molecule). New modes exert influence on the optical properties of an atom near 
a gap between spheres, and can be used to control the decay rates of an atom and molecule. From the other hand well pronounced dependencies of an atom optical properties on distance between spheres, dipole moment orientation and permittivity can be used for elaboration of new types of optical nanosensors.

Acknowledgement. The authors are grateful to the Russian Foundation for Basic Research (grant 04-02-16211) and the RAS Presidium program "The influence of atomic-crystalline and electron structure on the properties of condensed media" for the partial financial support of the present work.

\section{References}

[1] Raether, H. Surface Plasmons; Springer-Verlag: Berlin, 1998.

[2] Kreibig, U.; Vollmer, M. Optical Properties of Metal Clusters SpringerVerlag: Berlin, 1995.

[3] Hillenbrand, R.; Taubner, T.; Keilmann, F. Nature 2002, 418, 159.

[4] Moskovits,M.; Tay, L.-L.; Yang, J.; Haslett, T. Top. Appl. Phys. 2002, 82, 215.

[5] Wang, Z.; Pan, S.; Krauss, T.D.; Du, H.; Rothberg, L.J. Proc. Natl. Acad. Sci. USA 2003, 100, 8638.

[6] Nie, S.; Emory, S.R. Science 1997, 275, 1102.

[7] Michaels, A.M.; Nirmal, M.; Brus, L.E. J. Am. Chem. Soc. 1999, 121, 9932.

[8] Lakowicz, J.R.; Malicka, J.; Gryczynski, I.; Gryczynski, Z.; Geddes, C.D. J. Phys. D: Appl. Phys. 2003, 36, R240.

[9] Lakowicz, J.R.; Shen, B.; Gryczynski, Z.; D’Auria, S.; Gryczynski, I. Bichem. Biophys. Res. Commun. 2001, 286, 875.

[10] Guzatov, D.V.; Klimov, V.V. Chem. Phys. Lett. 2005, 412, 341.

[11] Brongersma, M.L. Nature Materials 2003, 2, 296.

[12] Haes, A.J.; Zou, S.L.; Schatz, G.C.; Van Duyne ,R.P. J. Phys. Chem. B 2004, 108, 6961.

[13] Haes, A.J.; Zou, S.L.; Schatz, G.C.; Van Duyne, R.P. J. Phys. Chem. B 2004, 108, 109.

[14] Su, K.-H.; Wei, Q.-H.; Zhang, X. Nano Lett. 2003, 3, 1087.

[15] Rechberger, W.; Hohenau, A.; Leitner, A.; Krenn, J.R.; Lamprecht, B.; Aussenegg, F.R. Optics Comm. 2003, 220, 137. 
[16] Tamaru, H.; Kuwata, H.; Miyazaki, H.T.; Miyano, K. Appl. Phys. Lett. textbf2002, 80, 1826.

[17] Prikulis, J.; Svedberg, F.; Kall, M.; Enger, J.; Ramser, K.; Goksor, M.; Hanstorp, D. Nano Lett. 2004, 4, 115.

[18] Nordlander P.; Oubre, C.; Prodan, E.; Li K.; Stockman, M.I. Nano Lett. 2004, 4, 899 .

[19] Paley, A.V.; Radchik, A.V.; Smith, G.B. J. Appl. Phys. 1993, 73, 3446.

[20] Pack, A.; Hietschold, M.; Wannemacher, R. Optics Commun. 2001, 194, 277.

[21] Xu, H.; Kall, M. Phys. Rev. Lett. 2002, 89, 246802.

[22] Genov, D.A.; Sarychev, A.K.; Shalaev, V.M.; Wei, A. Nano Lett. 2004, 4, 153.

[23] Claro, F. Phys. Rev. B 1982, 25, R7875.

[24] Ruppin, R. Phys. Rev. B 1982, 26, 3440.

[25] Olivares, I.; Rojas, R.; Claro, F. Phys. Rev. B 1987, 35, 2453.

[26] Ruppin, R. J. Phys. Soc. Japan 1989, 58, 1446.

[27] Chaumet, P.C.; Dufour, J.P. J. Electrostat. 1998, 43, 145.

[28] Stevenson, A.F. J. Appl. Phys. 1953, 24, 1134.

[29] Landau, L.D.; Lifshitz, E.M. Electrodynamics of Continuous Media; Pergamon Press: Oxford, 1971.

[30] Morse, P.M.; Feshbah, H. Methods of Theoretical Physics; McGraw-Hill: New York, 1953.

[31] Moon, P.; Spencer, D.E. Field Theory Handbook; Springer-Verlag: Berlin, 1988.

[32] Klimov, V.V.; Ducloy, M.; Letokhov, V.S. Quant. Electron. 2001, 31, 569.

[33] Klimov, V.V. Physics Uspekhi 2003, 46, 979.

[34] Klimov, V.V.; Guzatov, D.V. Spontaneous Emission of an Atom Placed Near Cluster of 2 Nanospheres (to be published).

[35] Engelbrecht, F.; Helbig, R. Phys. Rev. B 1993, 48, 15698. 


\section{List of Figure Captions}

Fig.1 Geometry of the problem.

Fig.2 Resonant permittivity as the function of normalized distance between two identical spheres $(m=1)$.

Fig.3 Surface charge density (in the relative units) for different modes with $m=1$ (the plane $y=0$ is shown). (a) $L$-modes; (b) $T$-modes; (c) $M$-modes. The distance between spheres is $\frac{R_{12}}{2 R_{0}}=1.1$. The broken line denotes a surface of the sphere; the colored lines correspond to the mode indices: red-1; green-2; blue-3 (cf. Fig.2).

Fig.4 The spontaneous emission radiative decay rate of an atom near the two nanospheres made of $\mathrm{SiC}$. The spheres have the radii of $50 \mathrm{~nm}$; the distance between their centers is of $101 \mathrm{~nm}$. The atom is placed precisely between the spheres and its dipole moment is directed: (a) transversally to the rotation axis; (b) longitudinally to the rotation axis. 


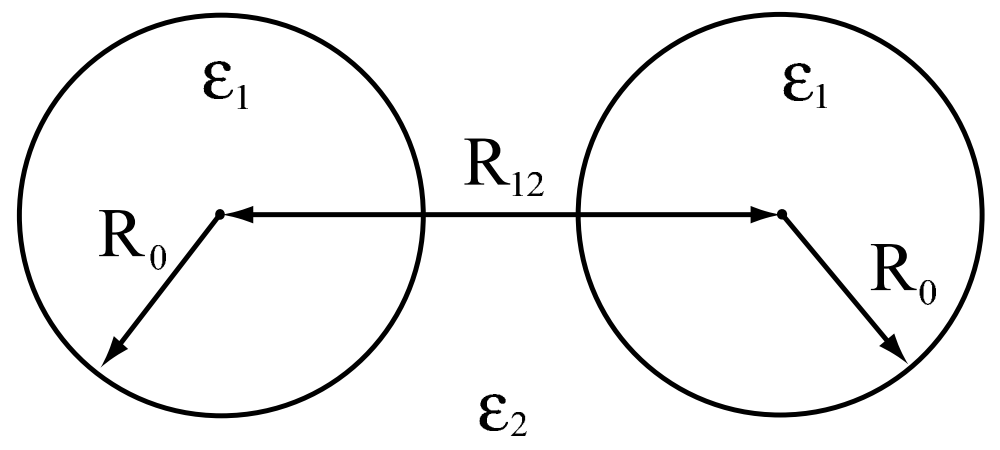

Figure 1:

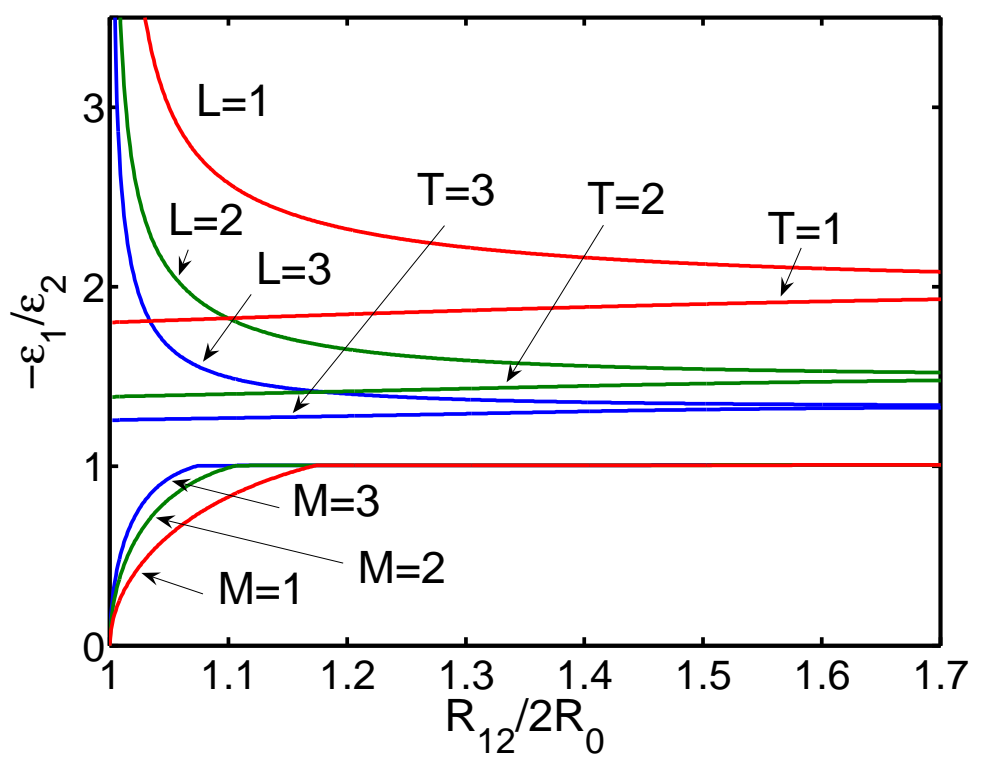

Figure 2: 

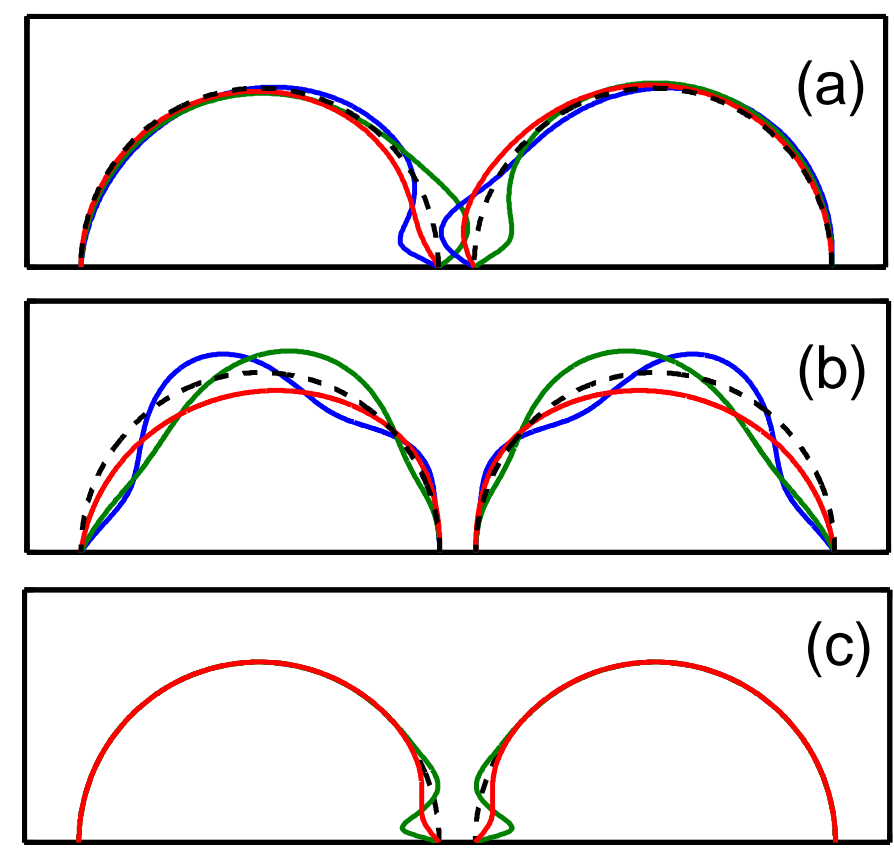

Figure 3: 

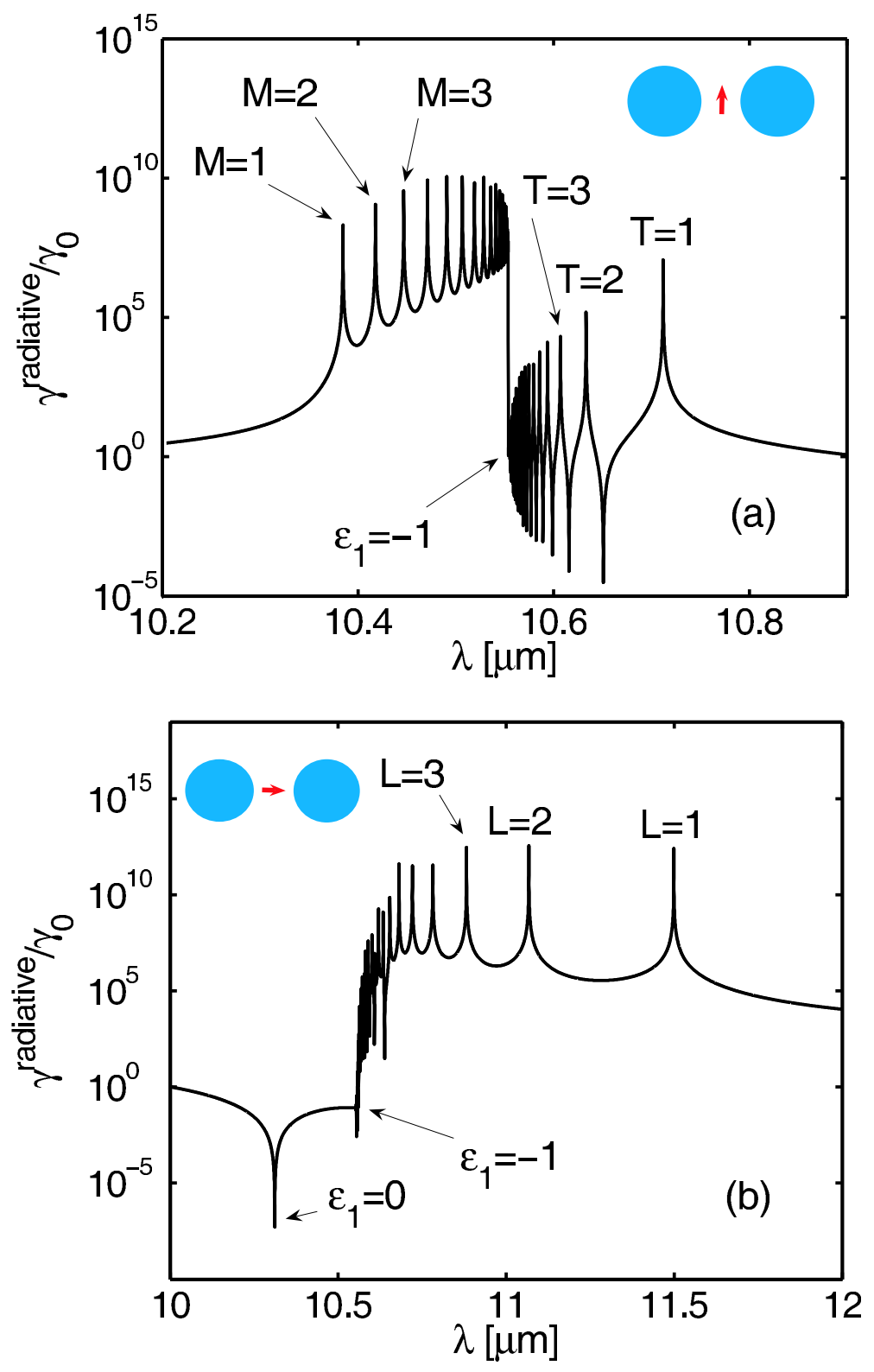

Figure 4: 